\title{
A visualized pulmonary arterial thrombus by using a new echocardiographic view in an intermediate-risk a cute pulmonary embolism patient: a case report
}

\section{Guanyu Mu}

Tianjin Medical University Second Hospital

\section{Feixue Li}

Tianjin Medical University Second Hospital

\section{Xiaolin Chen}

Tianjin Medical University Second Hospital

\section{Bo Zhao}

Tianjin Medical University Second Hospital

\section{Guangping Li}

Tianjin Medical University Second Hospital

\section{Huaying Fu ( $\nabla$ fuhuaying@tmu.edu.cn )}

Tianjin Medical University Second Hospital https://orcid.org/0000-0002-9392-1583

\section{Case report}

Keywords: Acute pulmonary embolism, new chocardiographic view, pulmonary artery clot

Posted Date: December 18th, 2019

DOl: https://doi.org/10.21203/rs.2.19164/v1

License: (c) (1) This work is licensed under a Creative Commons Attribution 4.0 International License. Read Full License 


\section{Abstract}

\section{Background}

Acute pulmonary embolism (APE) is a life-threatening disease with nonspecific clinical signs and symptoms. Rapid and accurate diagnosis is crucial for the clinical management of patients with acute pulmonary embolism. A new recommended echocardiography view may be of further help in the diagnosis, evaluate the change of the thrombosis and treatment effect.

\section{Case presentation}

We report a case of a 74-year-old man with a 12-day history of decreased exercise capacity and dyspnoea. The patient was diagnosed intermediate-risk APE as several pulmonary emboli in pulmonary artery were seen in multidetector computed tomographic pulmonary angiography with normal blood pressure and echocardiographic right ventricular overload. And we found a pulmonary artery clot in the right pulmonary artery through transthoracic echocardiography. After 11-days anticoagulation, the patient underwent a reassessment, showed decrease in RV diameter and pulmonary artery thrombus.

\section{Conclusion}

This case highlights the significant role that echocardiography played in a patient who presented pulmonary embolism with a stable hemodynamic situation and normal blood pressure. The new echocardiographic view could provide correct diagnoses by identifying the clot size and location visually. Knowledge of the echocardiography results of APE would aid the diagnosis.

\section{Background}

Venous thromboembolism clinically manifested as deep vein thrombosis or pulmonary embolism ${ }^{[1,2]}$. It is globally the third major cause of acute cardiovascular death ${ }^{[1,2]}$ as a major health problem. Rapid and accurate diagnosis is crucial for the clinical management of patients with acute pulmonary embolism $(\mathrm{APE})^{[3]}$. However, APE encompasses a wide clinical spectrum of severity ${ }^{[4-6]}$, ranging from asymptomatic disease to hemodynamic instability and shock. Due to the nonspecifific clinical signs and symptoms, a diagnosis of APE is frequently extremely challenging ${ }^{[5]}$.

In patients with suspected $\mathrm{PE}$, multidetector computed tomographic pulmonary angiography (CTPA) is the preferred method for imaging the pulmonary vasculature ${ }^{[1]}$. Echocardiography examination is not a routine diagnostic procedure in hemodynamic stable APE patients ${ }^{[1,7]}$, but might detect an increase in right ventricular wall thickness or a jet velocity of tricuspid insufficiency beyond values compatible with acute right ventricular pressure overload ${ }^{[1,8]}$. In addition to these indirect signs, emboli in the pulmonary artery may occasionally be found in certain nonstandard echocardiographic views ${ }^{[1]}$. Herein, we report 1 case of intermediate-risk APE patient with visualized pulmonary arterial thrombus by transthoracic echocardiography. 


\section{Case Presentation}

A 74-year-old man was admitted to our hospital with a 12-day history of decreased exercise capacity and dyspnoea. At presentation, he was afebrile with a blood pressure of $130 / 110 \mathrm{mmHg}$, heart rate of $80 / \mathrm{min}$ and respiratory rate of $20 / \mathrm{min}$. The physical examination was within normal limits. Electrocardiography showed sinus rhythm with first degree atrioventricular block, S wave in lead I, q wave and inverted T wave in lead III(Fig. 1). Laboratory evaluation was significant for elevated D-dimer of $4450.08 \mathrm{ng} / \mathrm{ml}$ and brain natriuretic peptide level of $4398 \mathrm{ng} / \mathrm{l}$. Complete blood count and coagulation studies were within normal limits. Tranthoracic echocardiography was performed. Right ventricular (RV) dysfunction was defined as dilatation of the right ventricle (right/left ventricle diameter ratio 1.2 in the apical four chamber view) combined with elevated systolic gradient through the tricuspid valve $(53 \mathrm{~mm} \mathrm{Hg})$, lack of relevant left ventricular (LV) dysfunction or valvular heart disease. Further evaluation with transthoracic echocardiography revealed a pulmonary artery clot of about $19.8 \mathrm{~mm} \star 32.2 \mathrm{~mm}$ seen at the right pulmonary artery, $21 \mathrm{~mm}$ from the bifurcation of the pulmonary artery (Fig. 2. Panels A and B, Video 1, 2). Meanwhile, venous Doppler studies of bilateral lower extremities showed right popliteal vein thrombosis. Computed tomography pulmonary angiography (CTPA) revealed several pulmonary emboli in right pulmonary artery (Panel C). The patient was diagnosed intermediate risk APE as systemic systolic blood pressure on admission $\geq 90 \mathrm{mmHg}$ with echocardiographic right ventricular overload and elevated serum levels of BNP. The patient was started on anticoagulation with low molecular weight heparin and oral warfarin. Tranthoracic echocardiography was re-evaluated after 11 days. The RV diameter was decreased combined with pulmonary artery thrombus decreased to $10.9 \mathrm{~mm} \star 13 \mathrm{~mm}$ (Panels D and E, Video 3, 4). The systolic gradient through the tricuspid valve was $43 \mathrm{~mm} \mathrm{Hg}$. The patient showed significant clinical improvement and was discharged home after being transitioned to oral warfarin.

\section{Discussion And Conclusion}

\section{Highlights}

It is very unusual to visualize a pulmonary artery thrombus on transthoracic echocardiography (TTE). This is the first case of a visualized pulmonary arterial thrombus in an intermediate-risk APE patient by transthoracic echocardiography. In this case report we demonstrate how transthoracic echocardiography permits to obtaining a new view, which allows real visualization of the moving thrombosis during the cardiac cycle. First, two dimensional echocardiograms of the parasternal long axis view were obtained. The right pulmonary artery view was obtained by placing the transducer inclination 30-45 degree to the chest wall at the second intercostal space immediately to the right of the sternum (Figure3.Panels A and B). Thus, we propose the new transthoracic echocardiography view as the tool of choice to evaluate the change of the thrombosis and treatment effect.

Role of Echocardiography in Evaluation of PE 
Echocardiography is not a diagnostic method for $\mathrm{PE}{ }^{[7]}$ but is used for patient risk stratification ${ }^{[1,9]}$. Both transesophageal echocardiography (TEE) and TTE provide noninvasive assessment of RV and LV size, systolic function, regional wall motion, valvular abnormalities, and hemodynamic assessment of filling pressure and right-heart pressures ${ }^{[10]}$. Thrombi in transit are rare to seen, and the appearance is typically that of a serpentine thrombus traversing the right-heart chambers ${ }^{[11-13]}$.

However, echocardiography has some inherent limitations in the diagnosis of APE. First, the ultrasound beam loses energy as travelling through tissue; therefore the structures away from the chest wall may not be well showed by TTE. Lower transducer frequency could improve penetration but decrease image resolution ${ }^{[14]}$. As a result, structures that may be important sources of embolism, such as the posteriorly located left atrium and its appendage, the interatrial septum, and the thoracic aorta, may be suboptimally visualized by TTE. Besides, it should be noted that signs of RV overload are not specific for PE and that acute and chronic RV abnormalities may be due to concomitant cardiac or respiratory disease $\mathrm{e}^{[1,15]}$, such as chronic obstructive pulmonary disease, obstructive sleep apnea, pulmonary hypertension, right-heart failure, and right-sided myocardial infarction, among others.

TTE is recommended for risk stratification in patients with PE (primarily for assessment of RV size and function $)^{[1]}$.It also may be considered in acutely ill, unstable patients in whom hemodynamically significant PE is suspected ${ }^{[13]}$.

\section{The new echocardiography view}

We propose the new transthoracic echocardiography view as the tool of choice to evaluate the change of the thrombosis and treatment effect. We recommend a new view which is right parasternal view and different with suprasternal view. It requires tilting the patient into a steep left lateral decubitus position. It provides the unique opportunity to view the ascending aorta and distal right pulmonary artery by placing the transducer inclination 30-45 degree to the chest wall at the second intercostal space immediately to the right of the sternum.

\section{Declarations}

\section{Ethics approval and consent to participate[}

This case report was approved by the Clinical Research Ethics Committee of the Second Hospital of Tianjin Medical University.

Consent for publication!

All the data in this case report, including any individual details, images or videos have consent for publication from the patient.

Availability of data and materials: 
Data sharing is not applicable to this article as no datasets were generated or analyzed during the current study.

\section{Competing interests:}

The authors declare that they have no competing interests.

\section{Funding:}

This study was funded by grants from Tianjin Natural Science Foundation (16JCYBJC25000), Key Laboratory Scientific research foundation of Second Hospital of Tianjin Medical University (2018ZDSYS03), Clinical study of Second Hospital of Tianjin Medical University (2019LC03) to H.F.

Authors' contributions:

GM and HF conceived of the case report and its design. FL, BZ and XC worked on acquisition of data and analysis. GM and HF drafted the manuscript. HF and GL revised the manuscript critically. All authors read and approved the final manuscript.

Acknowledgements:

Not applicable.

Authors' information:

Not applicable.

\section{Abbreviations}

APE: Acute pulmonary embolism;

PE: Pulmonary embolism;

CTPA: computed tomographic pulmonary angiography;

RV: Right ventricular;

LV: Left ventricular;

TTE: transthoracic echocardiography;

TEE: Transesophageal echocardiography

\section{References}


[1] Konstantinides SV, Meyer G, Becattini C, et al. 2019 ESC Guidelines for the diagnosis and management of acute pulmonary embolism developed in collaboration with the European Respiratory Society (ERS). Eur Heart J. 2019.

[2] Raskob GE, Angchaisuksiri P, Blanco AN, et al. Thrombosis: a major contributor to global disease burden. Arterioscler Thromb Vasc Biol. 2014. 34(11): 2363-71.

[3] Granér M, Harjola VP, Selander T, et al. N-terminal Pro-brain Natriuretic Peptide, High-sensitivity Troponin and Pulmonary Artery Clot Score as Predictors of Right Ventricular Dysfunction in Echocardiography. Heart Lung Circ. 2016. 25(6): 592-9.

[4] Dutta T, Frishman WH, Aronow WS. Echocardiography in the Evaluation of Pulmonary Embolism. Cardiol Rev. 2017. 25(6): 309-314.

[5] van der Hulle T, Dronkers CE, Klok FA, Huisman MV. Recent developments in the diagnosis and treatment of pulmonary embolism. J Intern Med. 2016. 279(1): 16-29.

[6] Stein PD, Beemath A, Matta F, et al. Clinical characteristics of patients with acute pulmonary embolism: data from PIOPED II. Am J Med. 2007. 120(10): 871-9.

[7] Roy PM, Colombet I, Durieux P, Chatellier G, Sors H, Meyer G. Systematic review and meta-analysis of strategies for the diagnosis of suspected pulmonary embolism. BMJ. 2005. 331(7511): 259.

[8] Guérin L, Couturaud F, Parent F, et al. Prevalence of chronic thromboembolic pulmonary hypertension after acute pulmonary embolism. Prevalence of CTEPH after pulmonary embolism. Thromb Haemost. 2014. 112(3): 598-605.

[9] Kurnicka K, Lichodziejewska B, Goliszek S, et al. Echocardiographic Pattern of Acute Pulmonary Embolism: Analysis of 511 Consecutive Patients. J Am Soc Echocardiogr. 2016. 29(9): 907-13.

[10] Surkova E, Muraru D, lliceto S, Badano LP. The use of multimodality cardiovascular imaging to assess right ventricular size and function. Int J Cardiol. 2016. 214: 54-69.

[11] Torbicki A, Galié N, Covezzoli A, Rossi E, De Rosa M, Goldhaber SZ. Right heart thrombi in pulmonary embolism: results from the International Cooperative Pulmonary Embolism Registry. J Am Coll Cardiol. 2003. 41(12): 2245-51.

[12] Koć M, Kostrubiec M, Elikowski W, et al. Outcome of patients with right heart thrombi: the Right Heart Thrombi European Registry. Eur Respir J. 2016. 47(3): 869-75.

[13] Barrios D, Rosa-Salazar V, Jiménez D, et al. Right heart thrombi in pulmonary embolism. Eur Respir J. 2016. 48(5): 1377-1385. 
[14] Mitchell C, Rahko PS, Blauwet LA, et al. Guidelines for Performing a Comprehensive Transthoracic Echocardiographic Examination in Adults: Recommendations from the American Society of Echocardiography. J Am Soc Echocardiogr. 2019. 32(1): 1-64.

[15] Bova C, Greco F, Misuraca G, et al. Diagnostic utility of echocardiography in patients with suspected pulmonary embolism. Am J Emerg Med. 2003. 21(3): 180-3.

\section{Figures}

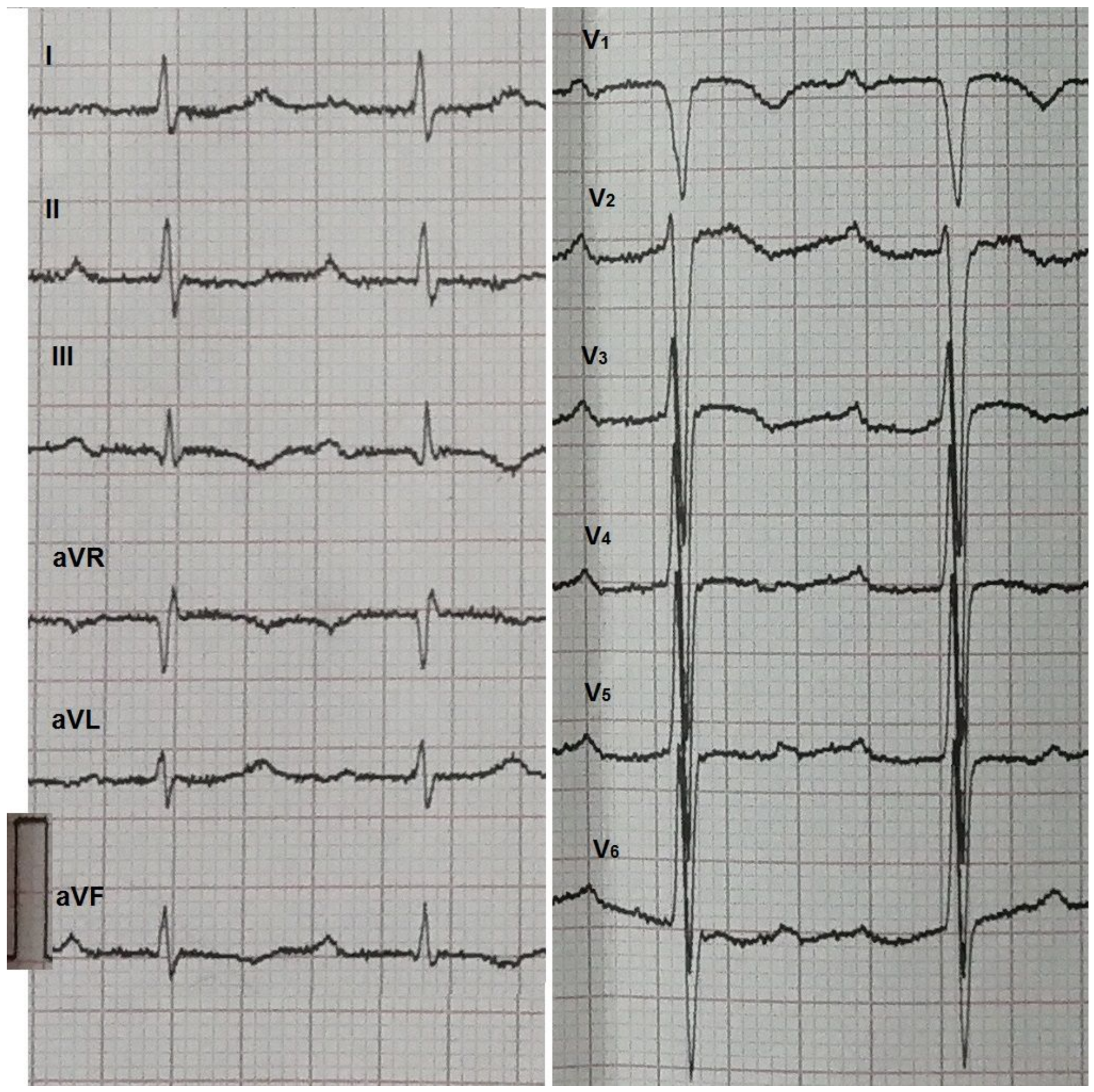


Figure 1

Electrocardiography of the patient. shows sinus rhythm with first degree atrioventricular block, $\mathrm{S}$ wave in lead I, q wave and inverted T wave in lead III.
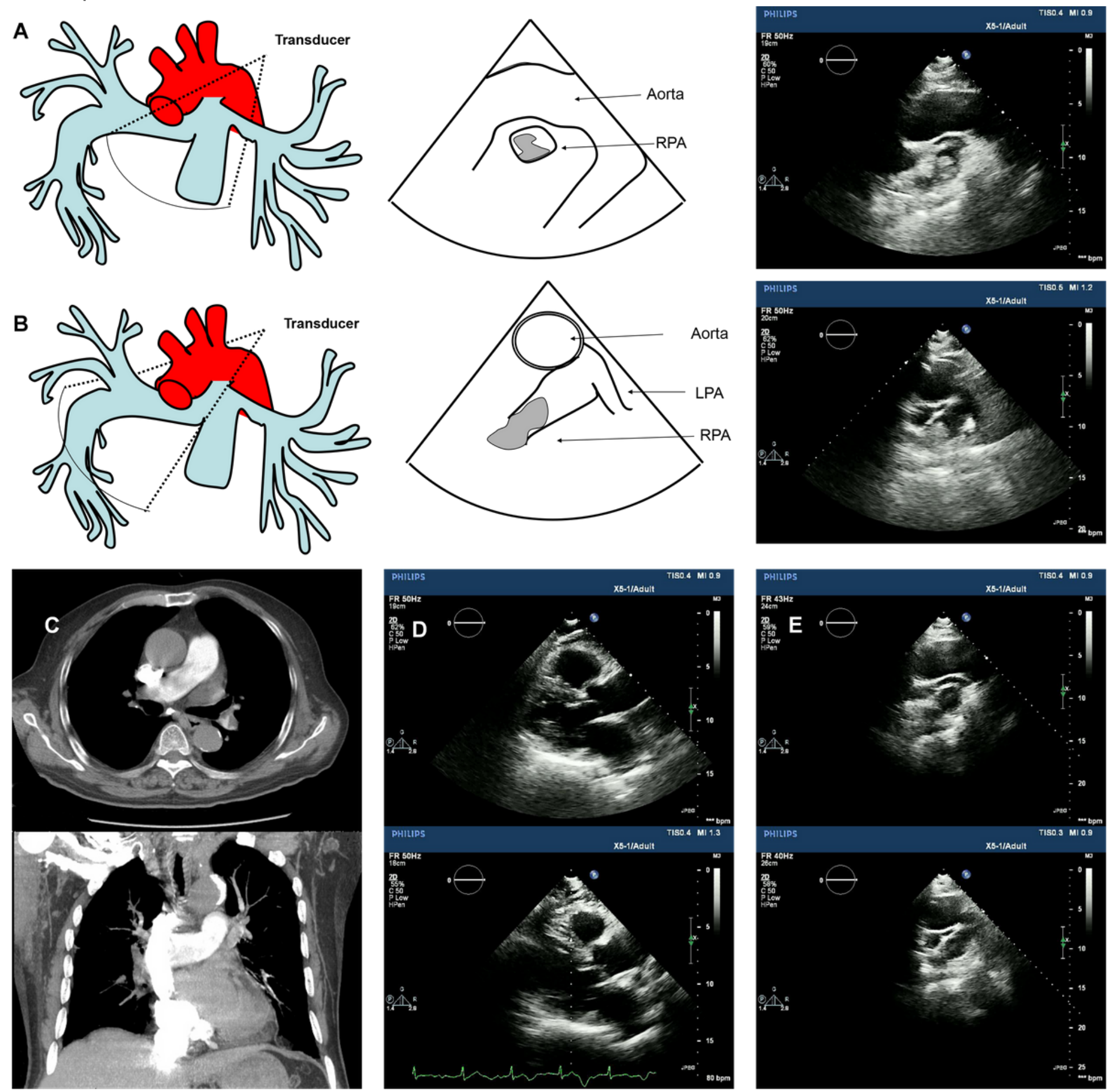

Figure 4

Imaging examinations of the patient. (A) Two-dimensional transthoracic echocardiographic image in an off-axis four-chamber view demonstrates a venous clot in transit (arrow) in the right atrium. Video 1 corresponds to (A). (B) Two-dimensional TTE at the level of pulmonary artery shows embolus (arrow) in 
the right PA (RPA). Video 2 corresponds to (B).(C)CTPA shows pulmonary emboli in right pulmonary artery from the transverse plane and the coronal plane respectively.(D) and (E) show decrease in RV diameter and pulmonary artery thrombus after 11-days treatment. Video 3 corresponds to (D).Video 4 corresponds to (E).Ao, Ascending aorta.

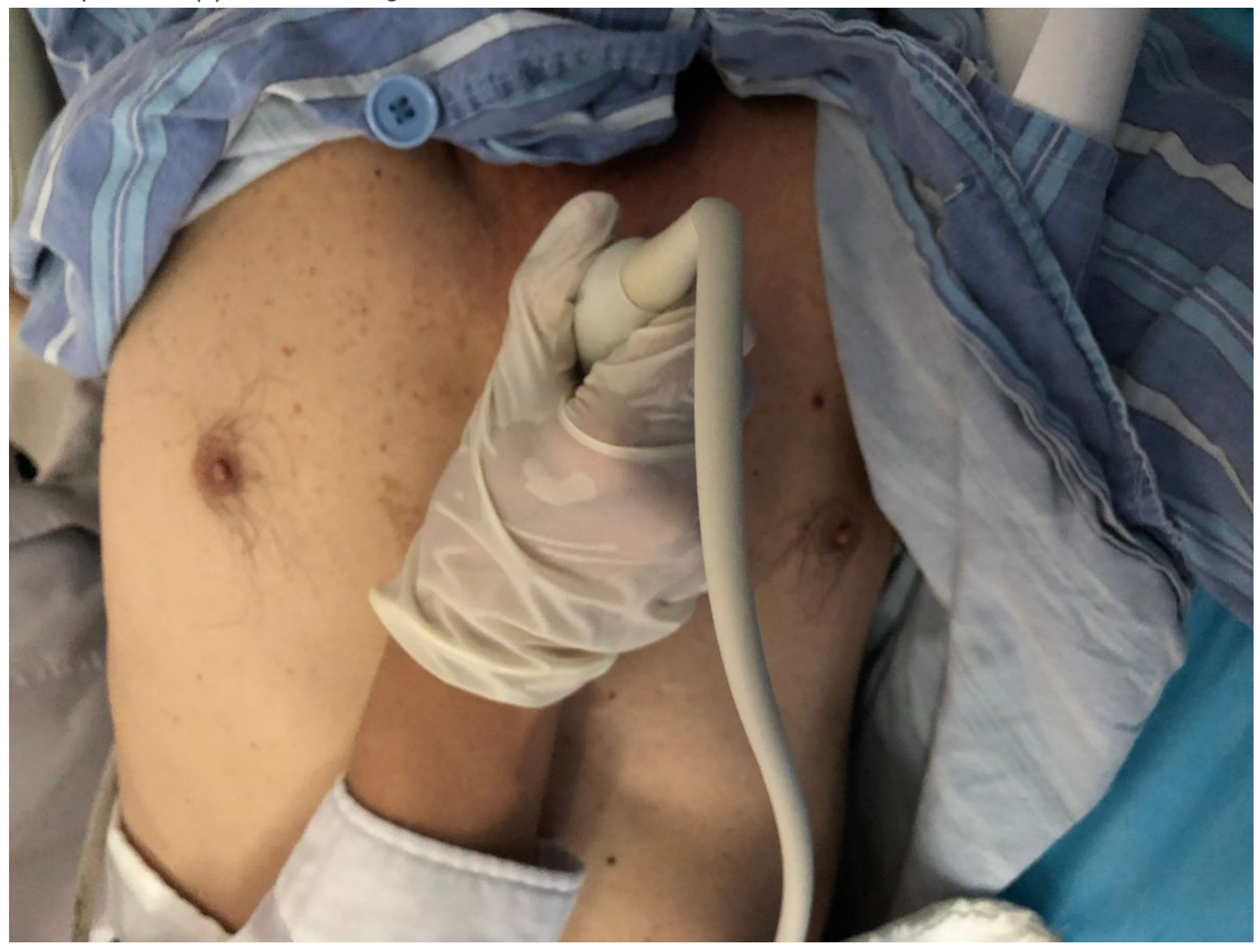

\section{Figure 5}

Acquirement of the new view.shows the transducer inclination 30-45 degree to the chest wall at the second intercostal space immediately to the right of the sternum.

\section{Supplementary Files}

This is a list of supplementary files associated with this preprint. Click to download.

- VIDE02.avi

- VIDE04.avi

- VIDEO3.avi 
- VIDE04.avi

- VIDE01.avi

- VIDE01.avi

- VIDE02.avi

- VIDE03.avi

Page 10/10 